\title{
A Large Posteriorly Located Prostatic Mass Lesion Challenging the Robotic Surgeon: Prostate Leiomyoma
}

\author{
Murat Keske, MD, Abdullah Erdem Canda, MD, Ali Fuat Atmaca, MD, Fevzi Bedir, MD, \\ Ilhan Gecit, MD, Arslan Ardicoglu, MD, Ozge Basaran Aydogdu, MD, \\ Yetkin Agackiran, MD, and Berrak Gumuskaya Ocal, $\mathrm{MD}^{6}$
}

\begin{abstract}
Background: Prostatic leiomyoma is a benign and rare condition of the prostate. Robotic surgery is increasingly being applied in the surgical management of prostate cancer.

Case Presentation: Herein, a mass lesion that was located in the posterior part of the prostate between seminal vesicles that was identified during robotic surgery is presented. This lesion further challenged the console surgeon during performing a robotic radical prostatectomy procedure for a $200 \mathrm{~g}$ large prostate with prostate cancer.

Conclusion: Prostatic leiomyomas that are benign mesenchymal smooth muscle tumors might present as a posteriorly located mass lesion between seminal vesicles that could challenge the surgeon during surgery, which should be kept in mind.
\end{abstract}

Keywords: prostate, leiomyoma, prostate cancer, robotic surgery

\section{Introduction}

$\mathbf{L}$ EIOMYOMA IS A benign tumor, which is made up of smooth muscle cells and located mostly in uterus, small bowel, and esophagus. Prostate leiomyoma is observed extremely rare. ${ }^{1}$

Herein, a prostatic leiomyoma that was pathologically reported after a robot-assisted radical prostatectomy (RARP) procedure is presented.

\section{Case Presentation}

A 70-year-old male patient with a serum prostate specific antigen value of $9.8 \mathrm{ng} / \mathrm{mL}$ underwent a transrectal ultrasound (TRUS) guided prostate biopsy. Pathology revealed prostatic adenocarcinoma with $3+3$ Gleason score in 1 core on the left side. A prostate volume of $190 \mathrm{cc}$ was measured by TRUS. No metastasis and lymphadenopathy were identified in abdominal CT and nuclear bone scan. Patient was referred to our institution who requested robotic surgical management.

The patient had a history of irritable bowel syndrome and had bilateral inguinal herniorrhaphy operation performed 8 years ago. In addition, he was on alpha blocker medication for obstructive urinary tract symptoms.

A transperitoneal RARP procedure with bilateral pelvic lymph node dissection was performed (by Dr. A.E.C.). Briefly, an incision was made on the anterior peritoneal covering of the Douglas' pouch, $\sim 1 \mathrm{~cm}$ proximal to its reflection on the rectum. Seminal vesicles (SVs) and vasa deferentia were dissected. At this stage, a $3 \times 3 \mathrm{~cm}$ sized posterior mass lesion

\footnotetext{
${ }^{1}$ Department of Urology, Ankara Ataturk Training and Research Hospital, Ankara, Turkey.

${ }^{2}$ Department of Urology, Ankara Ataturk Training and Research Hospital, Yildirim Beyazit University, School of Medicine, Ankara, Turkey.

${ }^{3}$ Department of Urology, Erzurum Training and Research Hospital, Erzurum, Turkey.

${ }^{4}$ Department of Urology, Inonu University, School of Medicine, Malatya, Turkey.

${ }_{5}^{5}$ Department of Pathology, Ankara Ataturk Training and Research Hospital, Ankara, Turkey.

${ }^{6}$ Department of Pathology, Ankara Ataturk Training and Research Hospital, Yildirim Beyazit University, School of Medicine, Ankara, Turkey.

(C) Murat Keske et al. 2017; Published by Mary Ann Liebert, Inc. This is an Open Access article distributed under the terms of the Creative Commons Attribution License, which permits unrestricted use, distribution, and reproduction in any medium, provided the original work is properly cited. Mary Ann Liebert, Inc. offers reprint services for those who want to order professionally produced copies of articles published under the Creative Commons Attribution (CC BY) license. To obtain a price quote, email Reprints@liebertpub.com. Please include the article's title or DOI, quantity, and delivery destination in your email.
} 


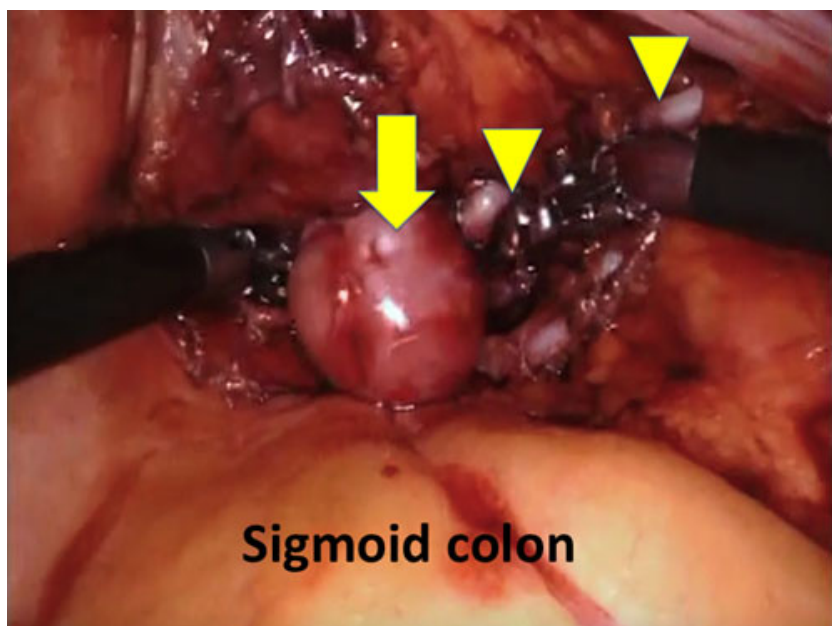

FIG. 1. Intraoperative picture showing a leiomyoma mass located between seminal vesicles. Arrow: leiomyoma mass, arrowheads: right vas deferens and right seminal vesicle.

protruding from the prostate between SVs was identified and dissected (Fig. 1). Then, Denonvilliers' fascia was opened. Rest of the RARP procedure was completed accordingly. Console time was 3 hours with estimated blood loss of $100 \mathrm{~mL}$. Figure 2 shows the removed specimen with a mass lesion protruding from the prostate located between SVs.

Postoperative follow-up was uneventful, abdominal drain was removed at the postoperative second day, and patient was discharged on the third day. Transurethral catheter was removed on postoperative day-10 followed by obtaining a cystography that demonstrated no leakage.

Pathology was reported as prostate adenocarcinoma, Gleason score $3+3$, tumor size of $5 \times 4 \times 3 \mathrm{~mm}$, left apex, and

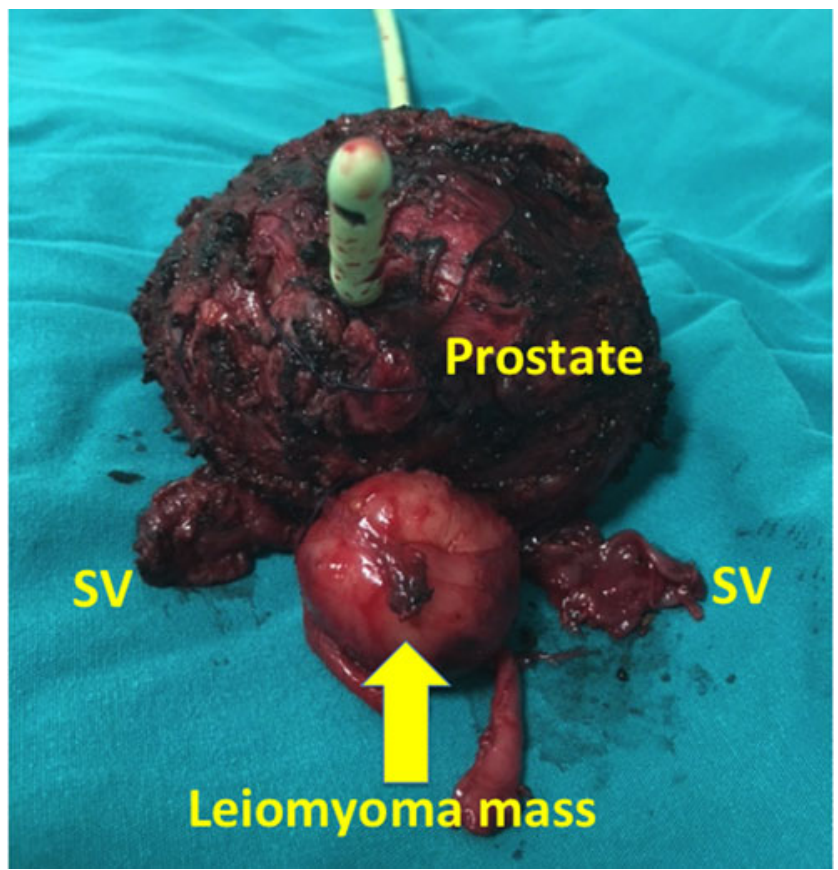

FIG. 2. Postoperative specimen showing leiomyoma located between seminal vesicles posteriorly. $\mathrm{SV}=$ seminal vesicle.

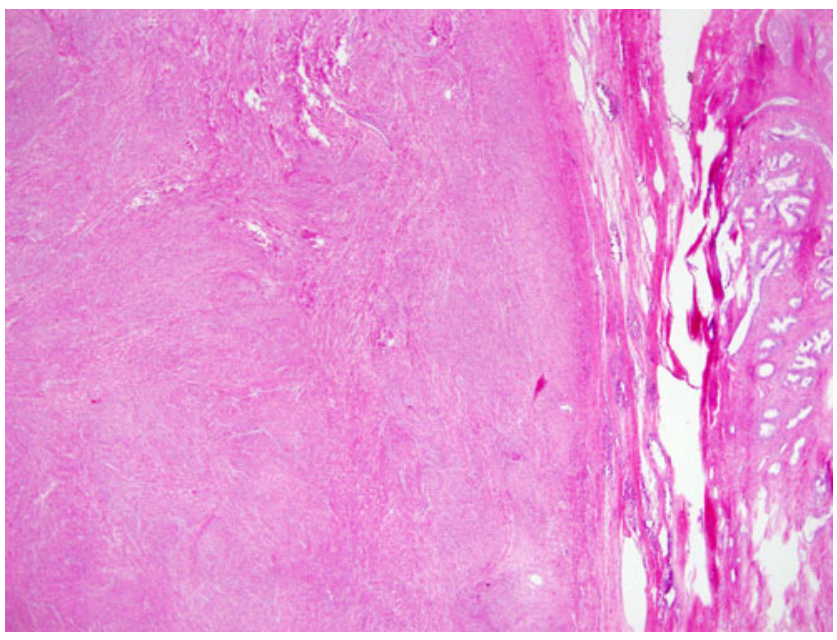

FIG. 3. A mass lesion, made up of smooth muscle bundles, is well demarcated from prostate tissue on the left $(H \& E \times 4) . H \& E=$ hematoxylin and eosin.

single focus with clear surgical margins. Posteriorly located mass pathology was reported as a leiomyoma with a size of $3.5 \times 3 \times 2.8 \mathrm{~cm}$. Prostate had a weight of $210 \mathrm{~g}$.

Microscopy of this mass lesion revealed a fascicular pattern of smooth muscle bundles separated by well-vascularized connective tissue (Fig. 3). Smooth muscle cells were elongated with eosinophilic or occasional fibrillar cytoplasm and distinct cell membranes. Lesion was noninfiltrative. One to 2 mitotic figures per 10 high power fields in most mitotically active area were observed, and there was no significant atypia (Fig. 4). Lesion was immunohistochemically positive with smooth muscle actin antibody (Fig. 4 inlet). Also a single focus of prostate adenocarcinoma with Gleason score $3+3$, size of $5 \times 4 \times 3 \mathrm{~mm}$, located on left apex, with clear surgical margins was reported.

At postoperative ninth-month follow-up, patient is fully urinary continent with no safety pad usage. He is able to have penile erection for sexual intercourse with use of phosphodiesterase type 5 inhibitors.

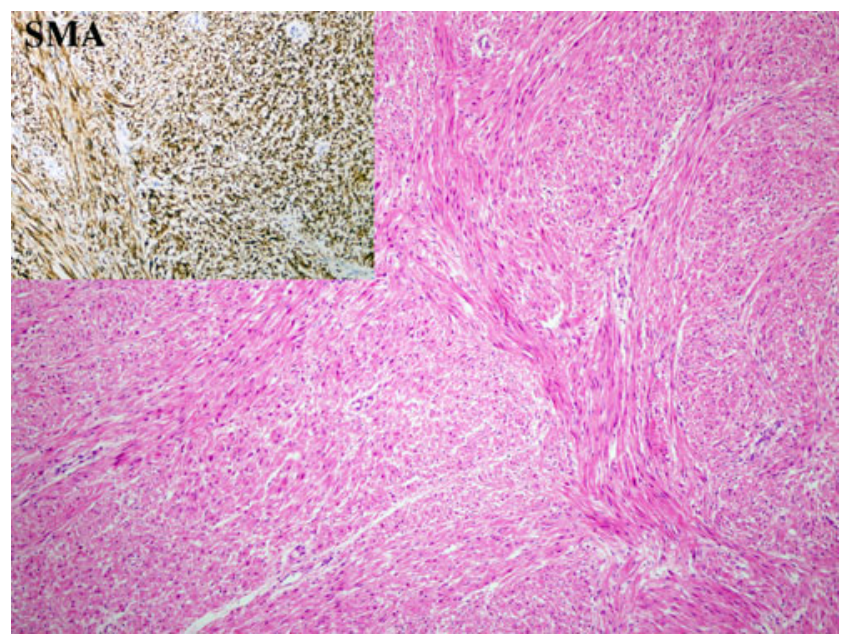

FIG. 4. Smooth muscle cells with bland cytology form fascicules $(\mathrm{H} \& \mathrm{E} \times 40)$. Immunohistochemically smooth muscle actin is positive (inset $\times 40$ ). 


\section{Discussion}

Although pure prostatic leiomyoma is rarely seen, it was reported initially in 1876 in the autopsy material by Lebel et al. ${ }^{2}$ In 1951, Kaufman and Berneike defined prostatic leiomyomas. ${ }^{3}$

In 2016, Kristensen et al. stated that prostatic leiomyomas were reported in $<100$ cases since it was defined. ${ }^{1}$ Chronic inflammation and infectious pathogens were suggested to be responsible for leiomyoma pathogenesis. In addition, the origin of prostatic leiomyoma may reveal as wastes of Müllerian duct. ${ }^{3}$ However, a certain mechanism of formation of prostatic leiomyoma is still not clearly presented.

Diagnosis of prostatic leiomyoma is generally made pathologic after surgery. Robotic surgery for the surgical management of a leiomyoma was previously performed by Aoun et al. that was only excision of the mass lesion rather than a complete RARP procedure. ${ }^{4}$ However, in our case the whole prostate with the posteriorly located leiomyoma was removed with the RARP procedure. The console surgeon in our case was challenged with the identification of a large mass lesion located between the SVs as this area has already a limited space. This mass was carefully dissected and freed from the surrounding structures. No serious adhesions were identified between the mass and its surroundings. Therefore, it was surgically not difficult to dissect and isolate this mass lesion. However, the presence of this mass lesion made it challenging to open the Denonvilliers' fascia due to its close proximity with rectum. At this stage, intra-abdominal pressure was elevated as much as $18 \mathrm{mmHg}$ temporarily to better see the area and $\mathrm{a} \times 4$ close up magnification was used with the da Vinci Xi surgical robot. Fourth arm of the robot holding a ProGrasp forceps was effectively used to lift up the prostate by holding from the SVs in an attempt to make the console surgeon to see the area below the mass lesion. It is important to keep in mind that this maneuver might lead to tearing off the SVs if too much force is applied as there is no tactile sensation in robotic surgery. A further trick that might also be applied could be using a $30^{\circ}$ up lens to better see the area below the mass lesion.

Leiomyoma is a benign tumor and has excellent prognosis when complete resection is applied and atypia is not detected. ${ }^{4}$ On our case, no atypia was reported related with the prostatic leiomyoma.

It was also reported that prostate leiomyosarcomas could rarely be diagnosed. ${ }^{5}$ Vandoros et al. stated that negative surgical margins and absence of metastatic disease at presentation were the only factors predictive of long-term survival. ${ }^{5}$ They also suggested multidisciplinary approach in the management of this condition. ${ }^{5}$ Therefore, prostatic leiomyosarcomas should also be kept in mind in the differential diagnosis.
In conclusion, prostatic leiomyomas that are benign mesenchymal smooth muscle tumors might present as a posteriorly located mass lesion between SVs that could challenge the surgeon during surgery, which should be kept in mind.

\section{Disclosure Statement}

No competing financial interests exist.

\section{References}

1. Kristensen VM, Loya AC, et al. Prostatic leiomyoma with bizarre nuclei: A case report. World J Nephrol Urol 2016;5:37-39.

2. Cohen MS, Mcdonald DF, Smith JH. Solitary leiomyoma of the prostate presenting as an encrusted intravesical mass. J Urol 1978;120:641-642.

3. Kaumann JJ, Berneike RR. Leiomyoma of the prostate. J Urol 1951;65:297-310.

4. Aoun F, de Saint Aubain Somerhausen N, van Velthoven R, et al. Prostate sparing robot-assisted laparoscopic treatment of leiomyoma of the prostate. J Robot Surg 2014;8:389-392.

5. Vandoros GP, Manolidis T, Karamouzis MV, et al. Leiomyosarcoma of the prostate: Case report and review of 54 previously published cases. Sarcoma 2008;2008:458709.

Address correspondence to: Murat Keske, $M D$ Department of Urology Ankara Ataturk Training and Research Hospital Ankara 06310 Turkey

E-mail: muratkeske@yahoo.co.uk

\begin{aligned} & \multicolumn{1}{c|}{ Abbreviations Used } \\ & $\mathrm{CT}=$ computed tomography \\ & RARP $=$ robot-assisted radical prostatectomy \\ & $\mathrm{SVS}=$ seminal vesicles \\ & TRUS $=$ transrectal ultrasound \end{aligned}

Cite this article as: Keske M, Canda AE, Atmaca AF, Bedir F, Gecit I, Ardicoglu A, Aydogdu OB, Agackiran Y, Ocal BG (2017) A large posteriorly located prostatic mass lesion challenging the robotic surgeon: prostate leiomyoma, Journal of Endourology Case Reports 3:1, 61-63, DOI: 10.1089/cren.2017.0033. 